\title{
Screening and Identification of Flocculant Producing Bacteria and Its Application in Wastewater
}

\author{
Sha Sha Zhang ${ }^{1}$, Ming $\mathrm{Li}^{2}$, Li Fang Wang ${ }^{2}$, Xiu Dong Wang ${ }^{1}$, Jian Feng Xu ${ }^{1, *}$ \\ ${ }^{1}$ Panjin Center for Inspection and Testing/Panjin CDC, Panjin, China \\ ${ }^{2}$ Heishan County Market Supervision Service Center, Jinzhou, China \\ Email address: \\ 250456075@qq.com (Sha Sha Zhang), xujianfeng69@126.com (Jian Feng Xu) \\ ${ }^{*}$ Corresponding author
}

\section{To cite this article:}

Sha Sha Zhang, Ming Li, Li Fang Wang, Xiu Dong Wang, Jian Feng Xu. Screening and Identification of Flocculant Producing Bacteria and Its Application in Wastewater. International Journal of Microbiology and Biotechnology. Vol. 6, No. 1, 2021, pp. 21-27. doi: $10.11648 /$ j.ijmb.20210601.13

Received: March 4, 2021; Accepted: March 17, 2021; Published: March 26, 2021

\begin{abstract}
Background: with the increasingly serious global water pollution, industrial and agricultural wastewater treatment is becoming more and more important. Flocculant precipitation is the first process in wastewater treatment. One of the keys to achieve efficient water purification is to add microbial flocculant with excellent performance. Objective: the research of high-efficiency and non-toxic microbial flocculant for wastewater treatment has increasingly become important in environmental protection. Method: CMC-Na medium and dilute iodine staining was used to separate and screen flocculant producing bacteria. The distribution of flocculant activity and the effect of different types of wastewater treatment were studied. Results: the flocculant producing strain 71-1 separate from ruminant feces was identified as Cellulosimicrobiums sp. The flocculating rate of MBF-P71 was 97.83\%, which had obvious degradation effect on COD value of pig farm manure water and ship port sewage. The removal rates of cod were $66.95 \%$ and $46.68 \%$ respectively. Conclusion: MBF-P71 has the advantages of good flocculation effect, low production cost, high efficiency and low dosage. It has a good development and application prospect for wastewater treatment.
\end{abstract}

Keywords: Microbial Flocculant, Cellulosimicrobiums, Screening and Identification, Sewage Purification, Flocculant-producing Strain, Wastewater

\section{Introduction}

Water is the source of human life. It is estimated that by 2025 , two thirds of the world population will probably live in lack of water resources. With the continuous growth of population and the development of urbanization in China, the demand for water resources keeps increasing, and the problem of water pollution is becoming increasingly prominent.

Microbial flocculant is a novel water treatment agent extracted from micro-organisms or their secretions by biotechnology and purified, which may be degraded naturally. It is also the key and core of flocculation technology for water treatment, and can be widely used in sewage or waste water treatment and sludge decontamination $[1,2]$.

In the natural environment, many microorganisms can be used as flocculant producing bacteria.
Microbial flocculant-producing bacteria are widely distributed in air, soil, ore, sewage, sludge and animals. They include fungi, bacteria and actinomycetes. The research on bacterial flocculant is relatively few, and the research on fungal flocculant is more than others. However, bacteria grow fast and have high practical significance in industrial production.

Microbial flocculant is high efficient, safe, non-toxic, and without secondary pollution. So it is promising for wide application. At present, a large amount of sewage is produced in China every year, and it is urgent to have an economical and simple treatment technology for industrial wastewater and domestic sewage treatment. For this, the study aims to explore the research and development concerning screening and identification of the strains for flocculant production, as well as application of microbial flocculants in degradation for different kinds of sewage or wastewater, so that the microbial flocculants could be used for kinds of sewage or wastewater 
efficiently and specifically, and the large-scale industrial production of flocculants become economic and feasible.

\section{Materials and Methods}

\subsection{Experimental Materials}

The strains were from the feces samples of ruminant collected from large-scale farms and slaughtering plants in three provinces of northeast China; the piggery liquid manure samples were from Wufengwang pig professional cooperative in Heishan, Jinzhou City, Liaoning Province.

China; domestic sewage samples were from Panjin City First Sewage Treatment Plant; food factory wastewater samples were from a Food Co. Ltd. of Panjin City; and marine ship sewage samples were from the ship that near the oceangoing shipsmoored at the port of Liaodong Bay, Panjin.

\subsection{Main Reagents}

Peptone, yeast extract and AGAR were purchased from OXOID, UK; sodium carboxymethyl cellulose from Beijing Solarbio Biotechnology co., LTD; Kaolin, potassium iodide, iodine, calcium chloride and other reagents from the Chemical Reagent Co., Ltd. of Sinopharm Group; API 20E, API 20NE and API 50CH kits for biochemical identification of bacteria and GP gram-positive bacteria identification card from BioMérieux; MALDI-TOF mass spectrum matrix and buffer from Brucker, Germany; bacterial genomic DNA extraction kit from Beijing Tiangen Biochemical Technology Co., Ltd.; $2 \times$ M5 HiPer plus Taq HiFi PCR mix from Mei5 Biotechnology Co. Ltd, Beijing; DL2000 DNA Marker from TaKaRa Biotechnology (Dalian) Co. Ltd., and agarose from Amresco Company.

\subsection{Instruments}

BG-270 water-jacket incubator (Medical Equipment Plant, Shanghai Boxun Industry \& Commerce Co. Ltd.), ZH-CHASL13 thermostatic oscillator (Jiangsu Photosynthesis Electromechanical Equipment Co. Ltd.), UV5200 spectrophotometer (Shanghai Yuanxi Instruments co., Ltd.), Allegra X-12R high-speed centrifuge (BECKMAN COULTER), GI 100DX autoclave (Xiamen Zhiwei Instruments Co., Ltd.), AC2-5S1 A2 model bio-safety cabinet (Esco Micro Pte Ltd, Singapore), NB-9 constant temperature magnetic stirrer (Suzhou JiulianTechnology Co., Ltd.), pH -100-a hand-held meter) (Zhejiang Lichen Technology Co., Ltd), matrix assisted laser desorption time-of-flight mass spectrometer (MALDI-TOF)(Brucker, Germany), automatic microbial identification system (Biomerieux, French), ET99731 COD/TOC multi-parameter comprehensive water quality tester (Lovibond, Germany), AND etc.

\subsection{Medium}

CMC-Na agar medium: $10 \mathrm{~g}$ of CMC-Na, $1 \mathrm{~g}$ of $\mathrm{K}_{2} \mathrm{HPO}_{4}$, $0.5 \mathrm{~g}$ of $\mathrm{Mg}_{2} \mathrm{SO}_{4}, 0.01 \mathrm{~g}$ of $\mathrm{Fe}_{2} \mathrm{SO}_{4}, 3 \mathrm{~g}$ of $\mathrm{NH}_{4} \mathrm{SO}_{4}, 15 \mathrm{~g}$ of agar, $1 \mathrm{~L}$ of water, pH 6.8-7.0, Enrichment medium: LB liquid medium.

\subsection{Methods}

\subsubsection{Enrichment and Initial Screening of Strains}

The samples of livestock feces were placed in $5 \mathrm{~mL}$ LB liquid medium and cultured at $37^{\circ} \mathrm{C}$ for $24-36 \mathrm{~h}$. CMC-Na agar was separated by the streak plate method and cultured at $37^{\circ} \mathrm{C}$ for $36-48 \mathrm{~h}$. A single colony of different morphology on the medium was selected for pure culture. The plate was dyed with a dye preparation of potassium iodide and dilute iodide solution for $1 \mathrm{~min}$, and a Vernier caliper was used for measuring the hydrolytic circle diameter of colony. The strains with high ratio of hydrolytic circle diameter to colony diameter were selected for rescreening [3].

\subsubsection{Strain Rescreening and Determination of Flocculation Activity}

The strains selected through initial screening were inoculated in LB liquid medium, and then incubated at $37^{\circ} \mathrm{C}$ in a shaker of $155 \mathrm{rpm}$ for $48 \mathrm{~h}$. The culture broth was centrifuged at $8000 \mathrm{r} / \mathrm{min}$ for $5 \mathrm{~min}$, the supernatant was discarded, and the precipitate was retained. Then the precipitate was resuspended in $0.1 \mathrm{M}$ PBS for washing twice, centrifuged at $8000 \mathrm{r} / \mathrm{min}$ for $5 \mathrm{~min}$, resuspended with PBS solution and broken through ultrasonication. The liquid obtained after $30 \mathrm{~min}$ of ultrasonic treatment was the microbial flocculant.

The flocculation activity is expressed by flocculation rate. Add $100 \mathrm{~mL}$ of $4 \mathrm{~g} / \mathrm{L}$ kaolin suspension, $1 \mathrm{~mL}$ of $10 \% \mathrm{CaCl}_{2}$ and $1 \mathrm{~mL}$ of culture medium in a $100 \mathrm{~mL}$ cylinder. Stir rapidly for $1 \mathrm{~min}$, slowly for $2 \mathrm{~min}$, and then hold for $10 \mathrm{~min}$. Then a spectrophotometer was used for measuring the absorbance of supernatant at $550 \mathrm{~nm}$. Meanwhile, distilled water instead of culture medium and $\mathrm{CaCl}_{2}$ solution was used as the control for experiment, and the following formula was used for calculating the flocculation rate:

$$
\text { Flocculation rate }=(\mathrm{A}-\mathrm{B}) / \mathrm{A} \times 100 \%
$$

where $A$ is absorbance value of the control group at $550 \mathrm{~nm}$, and $\mathrm{B}$ is the absorbance value of the test group at $550 \mathrm{~nm}$ [4].

\subsubsection{Identification of Strains}

Strains with high flocculation rate were selected by colony morphology observation, and a single pure colony was picked out for gram staining. MALDI-TOF MS was used for the identification of bacterial species, API 20E and API 20NE kits as well as GP gram-positive bacteria identification card were used for the identification of biochemical characteristics. Gene sequence analysis on strain 16S rDNA: About 3 to 5 single colonies were picked out and emulsified in $50 \mu \mathrm{L}$ of $0.25 \%$ sodium dodecyl sulfate $-0.05 \mathrm{~N} \mathrm{NaOH}$ solution, and incubated in metal bath at $100^{\circ} \mathrm{C}$ for $15 \mathrm{~min}$. Then $100 \mu \mathrm{L}$ of $\mathrm{H}_{2} \mathrm{O}$ was added to the mixture after incubation, and $2 \mu \mathrm{L}$ was taken and used for DNA template for PCR. PCR amplification conditions: primers (5'-GAGTTTGATCCTGGCTCAG-3'), 1510R (5'-GGCTACCTTGTTACGA-3')

The 16SrDNA of the isolates was performed PCR 
amplification with $25 \mu \mathrm{L}$ of PCR reaction system, including $12.5 \mu \mathrm{L}$ of $2 \times \mathrm{M} 5$ HiPer plus Taq HiFi PCR mix, $1.0 \mu \mathrm{L}$ of each primer, $2.0 \mu \mathrm{L}$ of template DNA, and $8.5 \mu \mathrm{L}$ of $\mathrm{ddH}_{2} \mathrm{O}$. PCR amplification conditions: $95^{\circ} \mathrm{C}$ for $5 \mathrm{~min}, 94^{\circ} \mathrm{C}$ for $45 \mathrm{~s}$, $52^{\circ} \mathrm{C}$ for $45 \mathrm{~s}, 72^{\circ} \mathrm{C}$ for $2 \mathrm{~min}$, and after 30 cycles, $72^{\circ} \mathrm{C}$ for 8 min. After $1.5 \%$ agarose gel electrophoresis, PCR products were sented to Sangon Biotech (Shanghai) Co., Ltd. for sequencing. The obtained gene sequences were uploaded to the Genbank database, and compared with those of standard strains for the BLAST sequence alignmenthomology. Clustal $\mathrm{X}$ software was used for multiple sequence alignments, and a phylogenetic tree was constructed by adjacency method of MEGA10.0 software. The confidence was tested through 1000 times of bootstrap analysis [5].

\subsubsection{Determination of Flocculation Distribution}

A certain amount of fermentation medium was centrifuged at $8000 \mathrm{r} / \mathrm{min}$ for $5 \mathrm{~min}$, dissolved in $0.1 \mathrm{~mL}$ PBS and resuspended for washing twice, and again centrifuged at 8000 $\mathrm{r} / \mathrm{min}$ for $5 \mathrm{~min}$. The precipitate was resuspended with PBS solution, broken through ultrasonication, and then diluted to volume of original fermentation broth with PBS. Then the flocculation rate of the fermentation supernatant, resuspended liquid and cell broken liquid were determined respectively.

\subsubsection{Application of Flocculants in the Treatment of Various Sewage and Wastewater}

For samples of piggery liquid manure, domestic sewage, food factory wastewater, and ship port sewage water, take 100 $\mathrm{mL}$ each, with $1.0 \mathrm{~mL}$ of $10 \% \mathrm{CaCl}_{2}$ solution, add in $1 \mathrm{~mL}$ of MBF-P71 cell wall broken liquid, shake well, and hold for 15 min for sedimentation. Then determine the flocculation rate, COD value and ammonia nitrogen content of samples.

\section{Results}

\subsection{Preliminary Screening Results}

Total 620 strains were isolated clinically, and preliminary screening by CMC-Na plate found 200 strains with relatively high cellulase activity. Table 1 showed 8 strains with the highest ratio of substrate hydrolytic circle diameter to colony diameter. As shown in Figure 1, dyeing with the dye preparation of potassium iodide and diluting iodide solution showed that strain 71-1 had a relative high hydrolysis capacity for cellulose, with obvious hydrolytic circle.

Table 1. Comparison of 8 strains of $\mathrm{CMC}-\mathrm{Na}$ substrate utilization and colony diameter.

\begin{tabular}{ll}
\hline Strain & $\begin{array}{l}\text { Substrate hydrolytic circle diameter to colony diameter } \\
\text { (average) }\end{array}$ \\
\hline P71-1 & $11.2 \pm 0.3$ \\
P85-1 & $8.6 \pm 0.2$ \\
P26-2 & $10.3 \pm 0.3$ \\
P40-2 & $27 \pm 0.3$ \\
P76-1 & $10.7 \pm 0.2$ \\
P99-1 & $11.1 \pm 0.1$ \\
P98-1 & $7.8 \pm 0.3$ \\
P72-3 & $8.1 \pm 0.2$ \\
\hline
\end{tabular}

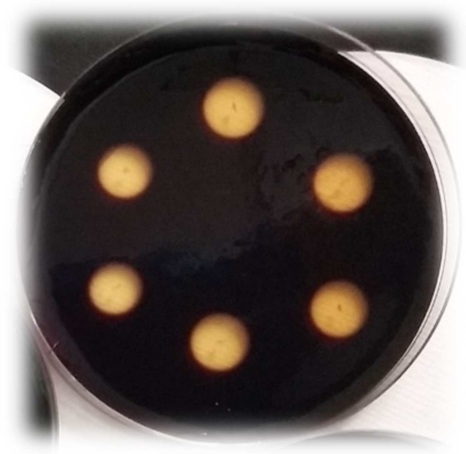

Figure 1. Cellulase hydrolysis circle of strain 71-1.

\subsection{Rescreening Results}

The 8 strains obtained through initial screening were respectively inoculated in LB liquid medium at $37^{\circ} \mathrm{C}$ for $48 \mathrm{~h}$, and then the flocculation activity of the culture medium was measured with kaolin suspension. Figure 2 showed the flocculation effect of liquid resuspended after cell walls were broken for some strains.

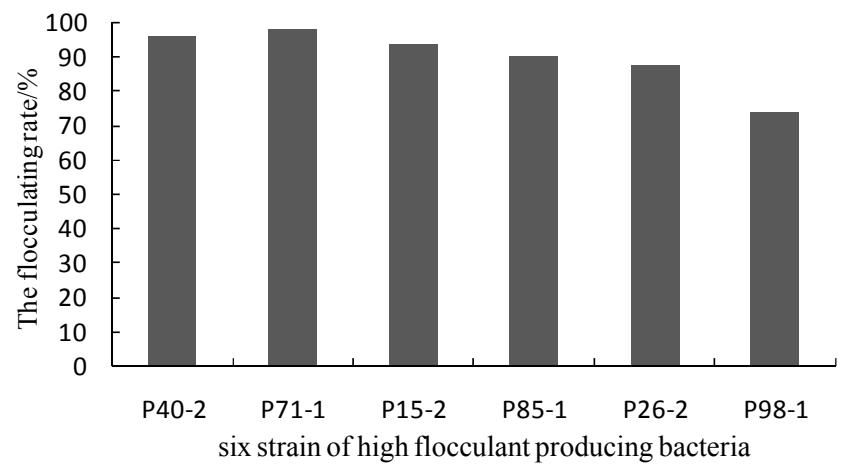

Figure 2. Flocculation effect of strains P40-2, P71-1, P15-2, P85-1, P26-2, P98-1.

\subsection{Identification Of strain 71-1}

\subsubsection{Morphological Identification}

Observed with naked eyes and under microscope, strain 71-1was yellow, round and slightly viscous on the LB plate, the surface was moist, smooth and opaque, with neat edge and a central bulge, diameter: $1-2 \mathrm{~mm}$. It was Gram positive, short rod-shaped, with obtuse ends, single or in pairs, without capsule and bud (Figure 3).
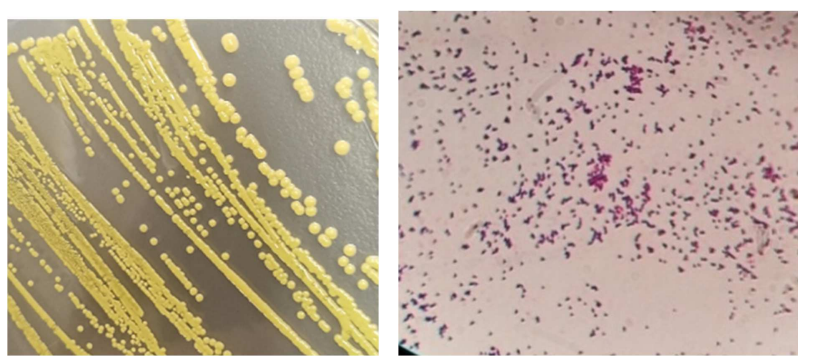

Figure 3. Colony morphology (A) and Gram staining morphology of isolated strains $(B)$ on LB solid medium. 


\subsubsection{Mass Spectrometry and Physiological or Biochemical Identification}

Strain 71-1 was identified by MALDI-TOF mass spectrometry (Figure 4). The biochemical identification results were shown in Table 2. It can be seen from Table 2 that strain 71-1 was partially different from the most approximate strain, Cellulosimicrobium funkei strain W6122 in biochemistry. Based on above morphological observation, mass spectrometry and biochemical identification results, the strain was preliminarily identified as Cellulosimicrobiums sp.

Table 2. Physiological and biochemical characteristics of bovine Cellulosimicrobium sp.

\begin{tabular}{|c|c|c|c|c|c|}
\hline \multirow{2}{*}{$\begin{array}{l}\text { Test } \\
\text { Item }\end{array}$} & \multicolumn{2}{|c|}{ Result } & \multirow{2}{*}{ Test Item } & \multicolumn{2}{|c|}{ Result } \\
\hline & $71-1$ & C.funkei W6122 & & 71-1 & C.funkei W6122 \\
\hline $\mathrm{NO}_{3}$ & + & - & TAD & + & - \\
\hline GLU & + & + & SOR & - & - \\
\hline $\mathrm{ADH}$ & - & - & RHA & - & - \\
\hline URE & - & - & SAC & + & - \\
\hline ESC & + & + & MEL & - & - \\
\hline GEL & - & + & AMY & - & - \\
\hline V-P & + & + & RIB & + & + \\
\hline ARA & + & - & LXYL & + & + \\
\hline MNE & + & + & $\mathrm{OX}$ & + & - \\
\hline MAN & - & - & XYL & + & + \\
\hline NAG & + & + & XLT & + & + \\
\hline MAL & + & + & RAF & - & + \\
\hline GNT & + & + & SAL & + & + \\
\hline ADI & - & - & CEL & + & + \\
\hline MLT & - & - & GNT & + & + \\
\hline CIT & - & - & ARL & + & - \\
\hline PAC & - & - & FUC & + & + \\
\hline ONPG & - & + & LYX & + & + \\
\hline GEN & + & + & LDC & - & + \\
\hline ODC & - & - & IND & + & + \\
\hline
\end{tabular}

\subsubsection{S rDNA Sequencing and Phylogenetic Analysis}

With DNA of strain 71-1 as a template, a single strip with fragment of $1377 \mathrm{bp}$ was obtained through PCR amplification (Figure 5), and $16 \mathrm{~s}$ rRNA gene sequence homology with Cellulosimicrobium funkei strain W6122 (NR 042937. 1) was 99.71\%. The serial number MN 901961 was obtained through uploading 16SrRNA sequence of strain 71-1 to NCBI. Through construction of phylogenetic tree, strain 71-1 and Cellulosimicrobium constitute a stable group of the closest relationship, and belong to the same evolutionary branch as a known standard strain, Cellulosimicrobium aquatile strain $3 \mathrm{bp}$, with sequence similarity of $99 \%$ (Figure 6). In combination with colony morphology, protein fingerprinting, $16 \mathrm{~S}$ rDNA identification results and physiological and biochemical characteristics, Strain 71-1 was identified as Cellulosimicrobiums $s p$. of effective species strain in Cellulosimicrobium.

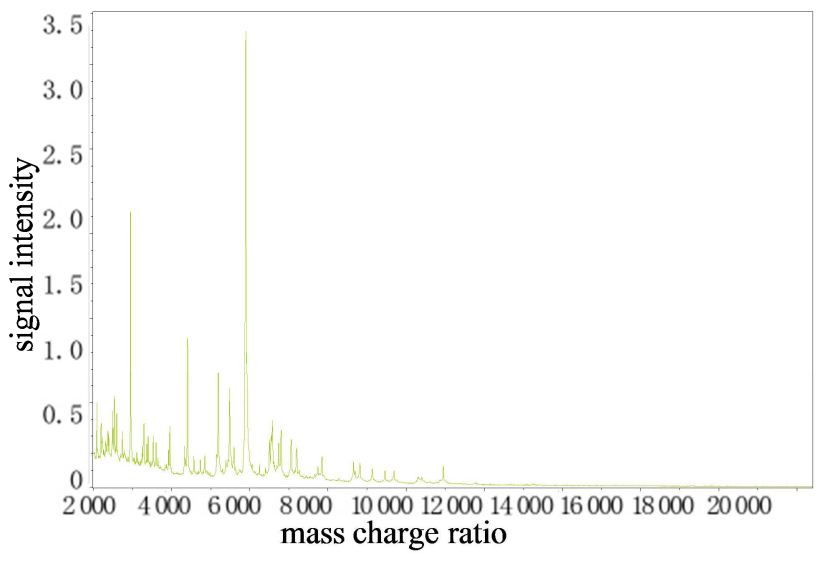

Figure 4. Protein fingerprinting of strain 71-1.

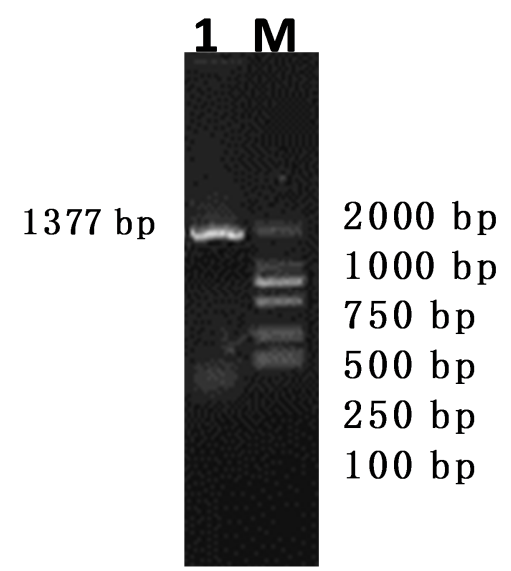

Figure 5. PCR amplification results of strain 71-1 16SrRNA.

\subsection{Flocculation Distribution of Strain 71-1}

The centrifuged fermentation broth, supernatant and bacterial suspension of strain 71-1 were taken for flocculation rate determination by kaolin test. The flocculation rate was $41.4 \%, 38.51 \%$ and $97.83 \%$, respectively (Figure 7). The flocculant prepared from the bacterial suspension of strain 71-1was named as MBF-P71.

\subsection{Application in Wastewater Treatment}

\subsubsection{MBF-P71 Treatment of Piggery Liquid Manure from Pig Farm}

MBF-P71 treatment of piggery liquid manure from pig farm and determination of flocculation rate, $\mathrm{COD}$ value and ammonia nitrogen. The test results showed that the flocculation rate reached $96.63 \%$. The COD value of piggery liquid manure from pig farm was $152 \mathrm{mg} / \mathrm{L}$ initially, and became $50.24 \mathrm{mg} / \mathrm{L}$ after MBF-P71 treatment. The COD removal rate was $66.95 \%$. The ammonia nitrogen of piggery liquid manure from pig farm was $135.25 \mathrm{mg} / \mathrm{L}$, and became $110.90 \mathrm{mg} / \mathrm{L}$ after treatment. The ammonia nitrogen removal rate was $18.0 \%$. 


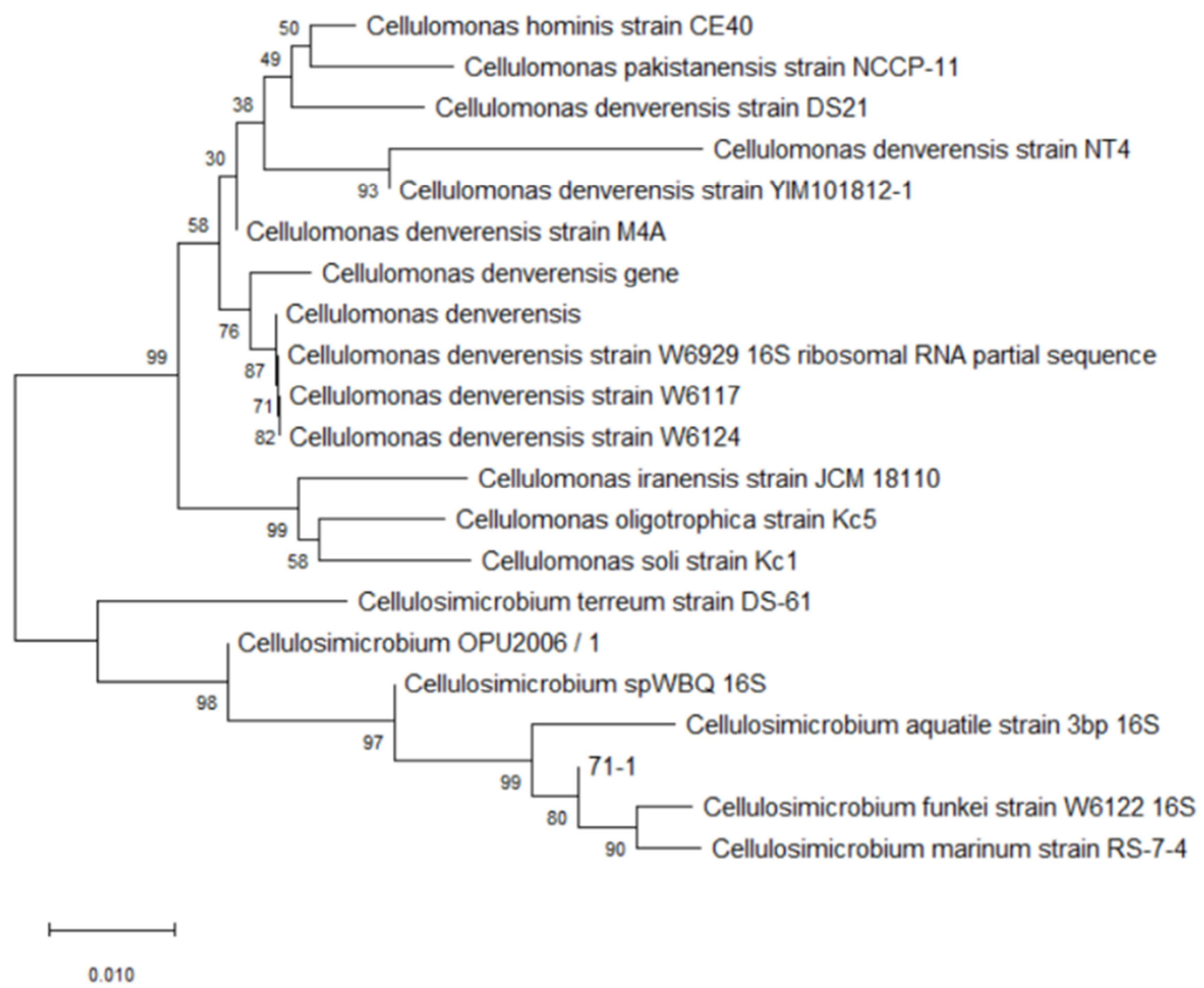

Figure 6. Phylogenetic tree of 16SrDNA gene of strain 71-1.

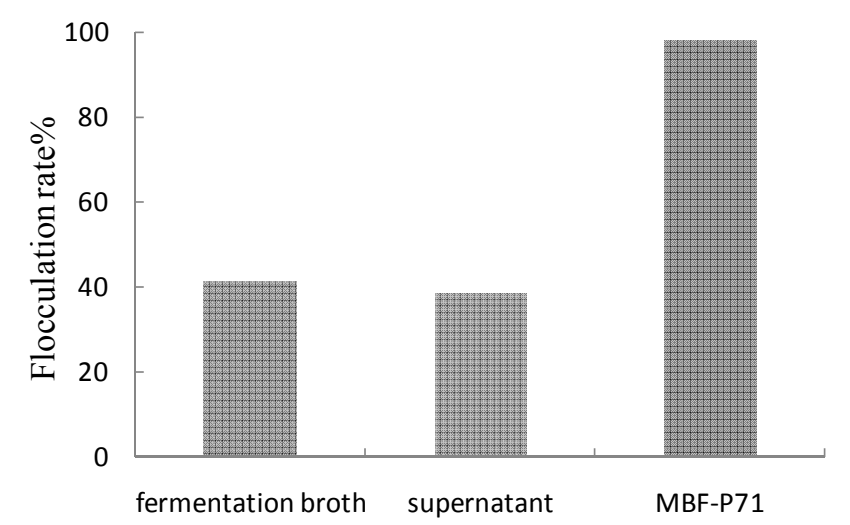

Figure 7. Distribution of flocculating active components in fermentation broth.

\subsubsection{MBF-P71 Treatment of Wastewater from Food Factory}

MBF-P71 treatment of wastewater discharged from bread factory and determination of flocculation rate, COD value and ammonia nitrogen. The test results showed that the flocculation rate reached $43.23 \%$. The COD value of bread factory wastewater was $8.09 \mathrm{~g} / \mathrm{L}$ initially, and became $7.41 \mathrm{~g} / \mathrm{L}$ after MBF-P71 treatment. The COD removal rate was $8.4 \%$. The ammonia nitrogen of bread factory wastewater was 23.5 $\mathrm{mg} / \mathrm{L}$ initially, and became $21.0 \mathrm{mg} / \mathrm{L}$ after MBF-P71 treatment, The ammonia nitrogen removal rate was $10.6 \%$.

\subsubsection{MBF-P71 Treatment of Domestic Sewage}

MBF-P71 treatment of domestic sewage from Panjin City First Sewage Treatment Plant and determination of flocculation rate, COD value and ammonia nitrogen. The test results showed that the flocculation rate reached $64.28 \%$. The COD value of untreated domestic sewage was $0.3 \mathrm{~g} / \mathrm{L}$, and after MBF-P71 treatment was $0.25 \mathrm{mg} / \mathrm{L}$. The COD removal rate was $16.7 \%$. The ammonia nitrogen of untreated domestic sewage was $1.3 \mathrm{mg} / \mathrm{L}$, and after MBF-P71 treatment was 1.2 $\mathrm{mg} / \mathrm{L}$. The ammonia nitrogen removal rate was $7.7 \%$.

\subsubsection{MBF-P71 Treatment of Ship Sewage}

For MBF-P71 treatment of sewage near shipsmoored at the port of Liaodong Bay, the flocculation rate was $56.73 \%$. The COD value of untreated port sewage was $4.82 \mathrm{mg} / \mathrm{L}$, and after MBF-P71 treatment was $2.57 \mathrm{mg} / \mathrm{L}$. The COD removal rate was $46.68 \%$. The ammonia nitrogen of untreated port sewage was $1.8 \mathrm{mg} / \mathrm{L}$, and after MBF-P71 treatment was $1.4 \mathrm{mg} / \mathrm{L}$. The ammonia nitrogen removal rate was $22.2 \%$.

\section{Discussion}

Since the 1980s, the research on microbial flocculants has 
been initiated comprehensively. In 1986, Kurane produced a protein flocculant named NOC-1 with Rhodococcus erythropolis S-1 isolated from nature. NOC-1 has a high and extensive flocculation activity, which may be used broadly with low cost, and it is the best microbial flocculant so far [6]. In 1997, the flocculant DP-152 was isolated from rod-shaped bacteria by Suh et al. who discovered that rod-shaped bacteria could also produce flocculant for the first time [7]. In this study, Cellulosimicrobium strains were screened out from a large number of ruminant samples. Cellulosimicrobium was established in 2001 by Schumann, which belongs to order of actinomycetes, suborder of micrococci, Cellulosimicrobium [8]. There are 6 effective phenotypic strains for Cellulosimicrobium, i.e. Cellulosimicrobium cellulans [9], Cellulosimicrobium aquatile sp, Cellulosimicrobium marinum $s p$, Cellulosimicrobium arenosum sp, Cellulosimicrobium funkei $s p$ and Cellulosimicrobium terreum sp. For the newly screened Strain 71-1, 16SrDNA sequencing and phylogenetic tree analysis were performed. The 16 s rRNA gene sequence homology with Cellulosimicrobium funkei strain W6122 (NR_042937. 1) was 99.71\%. It belongs to the same evolutionary branch as Cellulosimicrobium aquatile strain 3 bp, with sequence similarity of $99 \%$, and the same group as Cellulosimicrobium cellulans and Cellulosimicrobium marinum sp. But analyses on hydrolysis capacity for cellulose and flocculation showed that it might be a new strain 71-1 of Cellulosimicrobium aquatile strain $3 \mathrm{bp}$.

This study established a simple and rapid method for screening strains used for flocculant production. The initial screening was based on ratio of hydrolytic circle diameter to colony diameter. Total 200 strains were rapidly picked out from more than 600 strains for determination of flocculation rate by kaolin test, and the strains of high flocculation rate were selected for further application studies. The screening efficiency was significantly improved and the duration for strain screening was significantly shortened compared with traditional methods. An investigation on flocculation distribution of strain71-1 found that the flocculating rate of ultrasonicated liquid resuspended after centrifugation (97.83\%) was far higher than that of centrifuged fermentation supernatant (41.4\%) and liquid resuspended after centrifugation (38.51\%). This finding is quite different from the reported microbial flocculant prepared with centrifuged supernatant of fermentation liquid. Consulting a large number of domestic and foreign literatures found no report concerning microbial flocculant preparation with cell broken liquid. This finding provides reference for the studies on such microbial flocculant.

Chemical oxygen demand (COD) and ammonia nitrogen are important indicators for sewage treatment. A large amount of wastewater with high ammonia nitrogen and COD, if discharged into rivers, lakes or seas, will not only cause eutrophication or malodorous black of water, increase the difficulty and cost of water treatment, but even have toxic effects on people and organisms [10]. In 2010, Zhu Dan screened out Strain EH-5 from seabed mud, with which a microbial flocculantwas produced. The decrease rates of COD, chroma and turbidity of beer wastewater by the microbial flocculant were $64.15 \%, 69.57 \%$ and $95.91 \%$ respectively, and the decrease rates of COD, chroma and turbidity of dairy wastewater were $74.49 \%, 75.00 \%$ and $93.78 \%$ respectively [11]. In 2014, Ma Shuwen screened out from natural sediment of potato starch wastewater the mold and yeast with excellent flocculation performance, in which geotrichum candidum, after cultivation, made the removal rate of COD in the wastewater medium up to $90.32 \%$, and the removal rate of BOD5 up to $86.0 \%$ [12]. Yang Jinfeng treated tap water with microbial flocculant M-127, which showed that M-127 was superior to conventional flocculant in treatment efficiency, with water turbidity decrease rate of $93.89 \%$ at maximum [13]. The flocculant MBF-P71 developed in this study had different degradation effects on piggery liquid manure from pig farm, bread factory wastewater, domestic sewage and ship sewage. But in contrast, MBF-P71 has a higher capability in COD removal for piggery liquid manure from pig farm and ship sewage, and the removal rate was $66.95 \%$ and $46.68 \%$ respectively. The reduction of COD indicates the degradation of a large number of reducing substances such as organic pollutants in the water.

For this study, the next step for the flocculant MBF-P71 was to analyze active ingredients of MBF-P71, expand the application of MBF-P71 in combination with other biological agents for treatment of different kinds of wastewater or sewage $[14,15]$. The research, development and application of microbial flocculants are being oriented towards high efficiency, low cost and no pollution. With increasing awareness on environmental protection and ecological protection, the extensive application of microbial flocculants has brought practical benefits to production and life of humankind. In the long run, microbial flocculants are bound to become the hotspot for future development of flocculants in the aspect of wastewater or sewage treatment [16].

\section{Conclusion}

MBF-P71 produced by Cellulosimicrobium sp. separate from CMC-Na plate, the flocculation rate of MBF-P71 was $97.83 \%$. MBF-P71 may degrade piggery liquid manure from pig farm, wastewater discharged from bread factory, domestic sewage and ship sewage to different degrees, especially wasterwater from pig farm and ship sewage, the COD removal rate was $66.95 \%$ and $46.68 \%$ respectively.

\section{Author Contributions}

The authors whose names appear on the submission have contributed sufficiently to the scientific work and therefore share collective responsibility and accountability for the results. XJF and ZSS conceived the idea of the study; LM, WXD and WXF carried out the experiments and revised the manuscript.

\section{Funding}

This work was supported by a grant from Panjin CDC allocated to SS Zhang. The authors thank Jilin University Animal Medicine of College and Panjin Center for inspection 
and testing for the collaborative study conducted together.

\section{Data Availability}

No data have been fabricated or manipulated (including images) to support my conclusions. The 16S rRNA gene sequence of strain 71-1 were deposited in GenBank under accession numbers MN 901961.

\section{Conflict of Interest}

The authors declare that they have no conflict of interest.

\section{Ethical Statement}

I certify that this manuscript is original and has not been published and will not be submitted elsewhere for publication while being considered by SS Zhang. And the study is not split up into several parts to increase the quantity of submissions and submitted to various journals or to one journal over time. No data have been fabricated or manipulated to support our conclusions.

\section{References}

[1] Suresh A, Grygolowicz-Pawlak E, Pathak S, et al. (2018) Understanding and optimization of the flocculation process in biological wastewater treatment processes: A review. Chemosphere. 210: 401-416.

[2] Zhao C, Yang Q, Zhang H, et al. (2017) Optimization of Microbial Flocculant-Producing Medium for Bacillus subtilis. Indian J Microbiol. 57: 83-91.

[3] Michel Doumith, Carmen Buchrieser, Philippe Glaser, et al.(2004) Differentiation of the Major Listeria monocytogenes Serovars by Multiplex PCR. J. Clin. Microbiol., 42: 38193822 .

[4] ZHANG Xiang-mei, MA Tong-suo, ZHANG Hong-bing. (2006) Screening of a new flocculant producing strain and its characteristics. Industrial Water \& Wastewater, 37: 64-66.

[5] Fang Zhenhua, Zeng Yumei, Hong Meiling, et al. (2019) Isolation, identification and drug sensitivity test of pathogenic
Aeromonas Vickers from Chinese striped neck turtle. Chin. Animal Husbandry \& Vet. Medicine, 46: 2760-2768.

[6] Kurane R, Toeda K, Suzuki T. (1986) Culture conditions for production of microbial flocculant by Rhodococcus erythropolis. Agric. Biol. Chem. 50: 2309-2313.

[7] Suh H H, Kwon G S, Lee C H, et al. (1997) Characterization of bioflocculant produced by Bacillus sp. DP-152. Journal of Fermentation And Bioengineering 84: 108-112.

[8] SchumannP, Weiss N, Stackebrandt E. (2001) Reclassification of Cellulomonas cellulans (Stackebrandt and Keddie 1986) as Cellulosimicrobium cellulans gen. nov., comb. Nov. Int J Syst Evol Microbiol, 51: 1007-1010.

[9] Agunbiade MO, Van Heerden E, Pohl CH, et al. (2017) Flocculating performance of a bioflocculant produced by Arthrobacter humicola in sewage waste water treatment. BMC Biotechnol. 12; 17: 51.

[10] Mu J, Cui X, Shao M, Wang Y, Yang Q, Yang G, et al. (2019) Microbial origin of bioflocculation components within a promising natural bioflocculant resource of Ruditapes philippinarum conglutination mud from an aquaculture farm in Zhoushan, China. PLoS One. 19; 14: e0217679.

[11] Zhu Dan, Yang Lin, Su Hongyan. (2010) Isolation of Flocculant-producing Bacteria from Erhai Lake Sediment and Optimization of Culture Conditions. Journal of Dali university 09: 43-45.

[12] Ma Yiwen, Wang Mingming (2014) Study on flocculation of high-flocculation bacteria in potato starch wastewater. Food Industry Technology, 35: 170-173.

[13] Yang Jinfeng, Zhao Jihong, Song Feng-Hua (2009) Study on Decolorization of Dye Wastewater by Microbial Flocculant and Its Kinetics. Science \& Technology in Chemical Industry, 17: 16-19.

[14] Wang T, Tang X, Zhang S, et al. (2020) Roles of functional microbial flocculant in dyeing wastewater treatment: Bridging and adsorption. J Hazard Mater. 15; 384: 121506.

[15] Xia X, Lan S, Li X, et al. (2018) Characterization and coagulation-flocculation performance of a composite flocculant in high-turbidity drinking water treatment. Chemosphere. 206: 701-08.

[16] Ma Fang, Yang jixian. (2013) Compound Microbial Flocculant. Beijing: Science Press. 